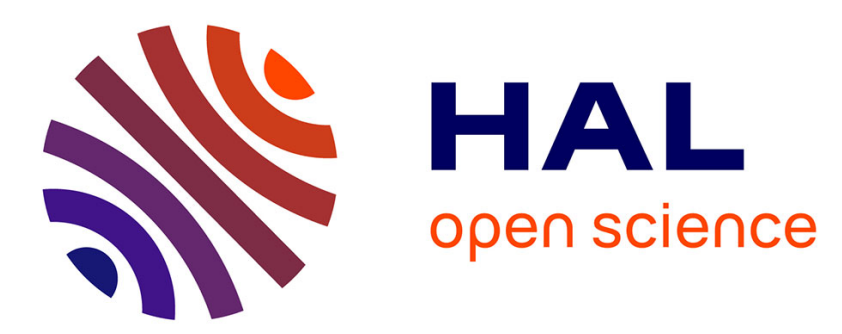

\title{
Exact level densities for the harmonic oscillator
}

P. van Isacker

\section{To cite this version:}

P. van Isacker. Exact level densities for the harmonic oscillator. Physical Review Letters, 2002, 89, pp.262502-1-262502-4. 10.1103/PhysRevLett.89.262502 . in2p3-00012283

HAL Id: in2p3-00012283

https://hal.in2p3.fr/in2p3-00012283

Submitted on 17 Jan 2003

HAL is a multi-disciplinary open access archive for the deposit and dissemination of scientific research documents, whether they are published or not. The documents may come from teaching and research institutions in France or abroad, or from public or private research centers.
L'archive ouverte pluridisciplinaire HAL, est destinée au dépôt et à la diffusion de documents scientifiques de niveau recherche, publiés ou non, émanant des établissements d'enseignement et de recherche français ou étrangers, des laboratoires publics ou privés. 


\title{
Exact level densities for the harmonic oscillator
}

\author{
P. Van Isacker \\ Grand Accélérateur National d'Ions Lourds, B.P. 55027, F-14076 Caen Cedex 5, France
}

\begin{abstract}
The number of levels of a many-fermion system confined by a harmonic oscillator potential is computed as a function of excitation energy. Due to its exact nature the formalism accounts for effects of shell structure on the level density. The method is easily extended to a variety of situations as is illustrated with the inclusion of isospin and deformation effects as well as a calculation of the number of spurious states.
\end{abstract}

A simple but commonly used estimate of the level density of a nucleus is known since the work of Bethe [1]. It assumes a gas of non-interacting fermions and, through a statistical analysis, arrives at the following expression for the level density (see also Chap. 2 of [2]):

$$
\rho(A, E)=\frac{1}{\sqrt{48} E} \exp \sqrt{2 \pi^{2} g\left(\epsilon_{\mathrm{F}}\right) E / 3}
$$

where $A$ is the particle number and $E$ is the excitation energy. The $A$-dependence in this formula arises through the density $g\left(\epsilon_{\mathrm{F}}\right)$ of single-particle levels at the Fermi-surface energy $\epsilon_{\mathrm{F}}$. The Fermi-gas estimate (1) cannot be reliably applied at low excitation energy $E$ where a low level density precludes a statistical analysis. At high excitation energy $E$, its derivation relies on the neglect of higher-order derivatives of the single-particle level density $g(\epsilon)$ and might be incorrect if this condition is not fulfilled [2]. Its most important limitation, however, is that it assumes independent particles.

After Bethe's work it was soon realized that his approach is an approximation to exact state-counting formulas [3]. With the advent of the nuclear shell model, effects of shell structure on nuclear densities were investigated in this way [4] and combinatorial counting 
techniques were used to evaluate the level density of Fermi systems consisting of equally spaced single-particle levels [5]. This approach continues to inspire research in this field (see, e.g. [6,7]). Nevertheless, formulas based on the statistical estimate (1) [and its extension to neutrons and protons-see Eq. (12) below] continue to be widely used [8] in applications where nuclear level densities are needed, e.g. in astrophysical studies [9]. A calculation of level densities that goes beyond the Fermi-gas model and takes account of the residual interactions between particles, is much more involved. Because of the rapidly increasing size of the model space with mass, a direct diagonalization of the Hamiltonian matrix quickly becomes impossible and other approaches must sought. One way to get at the level density is via spectral distribution methods and an analysis of the moments of the Hamiltonian [10]. More recently, level density distributions have been obtained in the context of the MonteCarlo shell model which also avoids diagonalization [11].

The approach presented in this Letter can be viewed as a counting method. A recurrence relation is established which allows the calculation of the exact number of harmonic-oscillator configurations with a given energy. The algorithm for computing such numbers can be established by relating them to those of a harmonic oscillator in a lower dimension.

Consider $A$ identical fermions with spin $s$ in an isotropic three-dimensional harmonic oscillator (HO). A single-particle state is labeled by three quantum numbers $n_{1}, n_{2}$, and $n_{3}$ which denote the numbers of oscillator quanta in the 1-, 2-, and 3-direction. Since the particles carry spin $s$, an additional quantum number $\sigma$ is needed which labels the spin degeneracy. A many-body Slater determinant is specified by the occupation numbers $k_{n_{1} n_{2} n_{3}}^{\sigma}$ with indices that run over all possible values (i.e. $n_{i}=0,1, \ldots$ and $2 s+1$ distinct values for $\sigma)$. For fermions, a single-particle level can be either occupied $\left(k_{n_{1} n_{2} n_{3}}^{\sigma}=1\right)$ or empty $\left(k_{n_{1} n_{2} n_{3}}^{\sigma}=0\right)$. The occupation numbers $k_{n_{1} n_{2} n_{3}}^{\sigma}$ of an $A$-particle state with total energy $E_{\mathbf{t}}$ must satisfy the following equations:

$$
\sum_{n_{1} n_{2} n_{3}=0}^{\infty} \sum_{\sigma} k_{n_{1} n_{2} n_{3}}^{\sigma}=A
$$




$$
\sum_{n_{1} n_{2} n_{3}=0}^{\infty} \sum_{\sigma}\left(n_{1}+n_{2}+n_{3}\right) k_{n_{1} n_{2} n_{3}}^{\sigma}=\mathcal{N}
$$

where $\mathcal{N}$ is the total number of oscillator quanta $\hbar \omega$ of the state, related with the total energy $E_{\mathbf{t}}$ through $E_{\mathbf{t}}=\left(\mathcal{N}+\frac{3}{2}\right) \hbar \omega$. The enumeration of all solutions $k_{n_{1} n_{2} n_{3}}^{\sigma}$ determines the number of distinct $A$-particle states which shall be denoted as $c_{3}(A, \mathcal{N})$ with the index 3 referring to the dimensionality of the $\mathrm{HO}$.

The derivation of the algorithm to calculate $c_{3}(A, \mathcal{N})$ requires elementary algebraic manipulations only and is as follows. Equation (2) can be rewritten as

$$
\begin{aligned}
\sum_{n_{1} n_{2}=0}^{\infty} \sum_{n_{3}=1}^{\infty} \sum_{\sigma} k_{n_{1} n_{2} n_{3}}^{\sigma} & =A-A^{\prime} \\
\sum_{n_{1} n_{2}=0}^{\infty} \sum_{n_{3}=1}^{\infty} \sum_{\sigma}\left(n_{1}+n_{2}+n_{3}\right) k_{n_{1} n_{2} n_{3}}^{\sigma} & =\mathcal{N}-\mathcal{N}^{\prime}
\end{aligned}
$$

where $A^{\prime}$ and $\mathcal{N}^{\prime}$ are defined through

$$
\begin{aligned}
\sum_{n_{1} n_{2}=0}^{\infty} \sum_{\sigma} k_{n_{1} n_{2} 0}^{\sigma} & =A^{\prime}, \\
\sum_{n_{1} n_{2}=0}^{\infty} \sum_{\sigma}\left(n_{1}+n_{2}\right) k_{n_{1} n_{2} 0}^{\sigma} & =\mathcal{N}^{\prime} .
\end{aligned}
$$

A new set of unknowns $k_{n_{1} n_{2} n_{3}}^{\sigma} \equiv k_{n_{1} n_{2} n_{3}+1}^{\sigma}$ is now introduced. The sums in (3) have again $n_{3}$ running from 0 to $\infty$ in terms of the new unknowns and by subtraction of the first from the second equation one finds

$$
\begin{gathered}
\sum_{n_{1} n_{2} n_{3}=0}^{\infty} \sum_{\sigma} k_{n_{1} n_{2} n_{3}}^{\prime \sigma}=A-A^{\prime} \\
\sum_{n_{1} n_{2} n_{3}=0}^{\infty} \sum_{\sigma}\left(n_{1}+n_{2}+n_{3}\right) k_{n_{1} n_{2} n_{3}}^{\prime \sigma}
\end{gathered}
$$

Equation (5) has a structure identical to the original one but with smaller numbers of particles and quanta; Eq. (4) is also of a similar type but for a two-dimensional HO. In fact, the entire derivation can be carried out for a $d$-dimensional $\mathrm{HO}$ and yields the following recurrence relation:

$$
c_{d}(A, \mathcal{N})=\sum_{A^{\prime} \mathcal{N}^{\prime}} c_{d-1}\left(A^{\prime}, \mathcal{N}^{\prime}\right) c_{d}\left(A-A^{\prime}, \mathcal{N}-\mathcal{N}^{\prime}-A+A^{\prime}\right)
$$


One needs, in addition, some boundary values for $c_{d}(A, \mathcal{N})$. They are:

$$
\begin{aligned}
c_{d}(A=0, \mathcal{N}) & =\delta_{\mathcal{N} 0}, \\
c_{d}(A, \mathcal{N}) & =0, \quad \text { if } \quad \mathcal{N}<\mathcal{M}_{d}^{A},
\end{aligned}
$$

where $\mathcal{M}_{d}^{A}$ denotes the minimum number of quanta for $A$ particles in a $d$-dimensional HO. The first condition expresses the existence of a single vacuum state and the second states that a minimum number of quanta is required for constructing a Pauli-allowed $A$-particle state. Note that the dependence on the spin degeneracy is introduced in the boundary values only, in particular, via the minimum number of quanta $\mathcal{M}_{d}^{A}$. Finally, an analysis of the one-dimensional case shows that the same recurrence relation (6) remains valid provided one takes for $d=0$

$$
c_{0}(A, \mathcal{N})=\frac{(2 s+1) !}{A !(2 s+1-A) !} \delta_{\mathcal{N} 0}
$$

The algorithm (6) is easily implemented numerically through a recursive procedure. An illustration of it is given in Fig. 1 which shows the level density $\rho(A, E)$ for 70 particles (a closed-shell number for the three-dimensional HO with $\operatorname{spin} s=\frac{1}{2}$ ) up to an excitation energy of $30 \hbar \omega$. The dots are calculated from $\rho(A, E) \equiv c_{3}\left(A, \mathcal{N}=\mathcal{M}_{3}^{A}+E / \hbar \omega\right) / \hbar \omega$ for discrete values $E=0, \hbar \omega, 2 \hbar \omega, \ldots$ and with $\mathcal{M}_{3}^{70}=210$, the minimum number of oscillator quanta for $70 s=\frac{1}{2}$ particles. The curve shows the Fermi-gas result (1) with the HO single-particle level density

$$
g(\epsilon)=\frac{1}{2}(2 s+1)(N+1)(N+2), \quad \epsilon=N \hbar \omega,
$$

with $N$ the major-shell quantum number. For $A=70$ particles, the Fermi energy corresponds to $N=4, g\left(\epsilon_{\mathrm{F}}\right)=15(2 s+1)$. Deviations from the statistical treatment occur at low energy but also at high energy because the dependence of $g(\epsilon)$ on $\epsilon$ is quadratic. Note that the number of configurations is huge but is nevertheless given exactly by (6); for example, the number of independent Slater determinants for $70 s=\frac{1}{2}$ particles at an excitation energy of $30 \hbar \omega$ is 896647829312727644544457613187541 . 
The recurrence relation (6) defines the problem in the simplest possible situation of identical particles in an isotropic oscillator. The advantage of the formalism is that it can be easily adapted to a variety of situations as will be illustrated with some examples.

A first application concerns the enumeration of spurious states. To estimate nuclear level densities, one is interested only in states that are in the ground configuration with respect to the center-of-mass excitation while the solutions of (2) include non-physical ones which represent a collective translation of the nucleus as a whole. Let us denote the number of physical solutions of (2) as $\tilde{c}_{3}\left(A, \mathcal{N}_{\mathrm{e}}\right)$ where $\mathcal{N}_{\mathrm{e}} \equiv \mathcal{N}-\mathcal{M}_{3}^{A}$ is the number of HO quanta above the minimum required for $A$ particles. The number $\tilde{c}_{3}\left(A, \mathcal{N}_{\mathrm{e}}\right)$ is found by substracting from the total number those that can be constructed by acting (possibly several times) with the step-up operator for the center-of-mass motion, $B_{\mu}^{\dagger} \equiv \sum_{i} b_{\mu}^{\dagger}(i) / \sqrt{A}$, on any physical state (see Chap. 4 of [12]). Hence

$$
\tilde{c}_{3}\left(A, \mathcal{N}_{\mathrm{e}}\right)=c_{3}(A, \mathcal{N})-\sum_{\mathcal{N}_{\mathrm{e}}^{\prime}=1}^{\mathcal{N}_{\mathrm{e}}} \frac{1}{2}\left(\mathcal{N}_{\mathrm{e}}^{\prime}+1\right)\left(\mathcal{N}_{\mathrm{e}}^{\prime}+2\right) \tilde{c}_{3}\left(A, \mathcal{N}_{\mathrm{e}}-\mathcal{N}_{\mathrm{e}}^{\prime}\right)
$$

This recurrence relation allows an easy determination of the fraction of spurious states, $1-\tilde{c}_{3} / c_{3}$, and is illustrated in Fig. 1 for $A=70$. The percentage of spurious states increases from $0 \%$ for $\mathcal{N}_{\mathrm{e}}=0$ (as should be) to $58.34 \%$ for $\mathcal{N}_{\mathrm{e}}=30$.

An important generalization concerns systems consisting of two different types of fermions, e.g. nuclei with neutrons and protons. The occupation numbers in this case are $k_{n_{1} n_{2} n_{3}}^{\sigma \tau}$, where $\tau$ is the intrinsic label of the fermion, say $\tau=+$ for a neutron and $\tau=-$ for a proton. The number of possible configurations for $N$ neutrons, $Z$ protons, and $\mathcal{N}$ quanta, $c_{3}(N, Z, \mathcal{N})$, can again be obtained with recursive techniques. Alternatively, it follows from

$$
c_{3}(N, Z, \mathcal{N})=\sum_{\mathcal{N}^{\prime}} c_{3}\left(N, \mathcal{N}-\mathcal{N}^{\prime}\right) c_{3}(Z, \mathcal{N})
$$

The quantities $c_{3}(N, Z, \mathcal{N})$ can be evaluated for closed as well as for open shells and this provides a simple way for estimating the effect of shell structure on nuclear level densities. This is illustrated in Fig. 2 which shows the exact $\mathrm{HO}$ results for systems corresponding to the nuclei ${ }^{16} \mathrm{O}$ and ${ }^{28} \mathrm{Si}$ and compares them to the Fermi-gas estimate $[1,2]$ 


$$
\rho(N, Z, E)=\frac{\sqrt[4]{6}}{12} g_{0} \sqrt{\frac{g_{0}^{2}}{4 g_{\mathrm{n}}\left(\epsilon_{\mathrm{F}}^{\mathrm{n}}\right) g_{\mathrm{p}}\left(\epsilon_{\mathrm{F}}^{\mathrm{p}}\right)}}\left(g_{0} E\right)^{-5 / 4} \exp \sqrt{2 \pi^{2} g_{0} E / 3},
$$

where $g_{0}$ is the sum of the densities of single-neutron and single-proton levels at their respective Fermi-surface energies, $g_{0}=g_{\mathrm{n}}\left(\epsilon_{\mathrm{F}}^{\mathrm{n}}\right)+g_{\mathrm{p}}\left(\epsilon_{\mathrm{F}}^{\mathrm{p}}\right)$. The single-particle level densities are obtained from a linear interpolation in (9) of the major-shell quantum number $N$ between ${ }^{16} \mathrm{O}(N=2)$ and ${ }^{40} \mathrm{Ca}(N=3)$. It is clear from Fig. 2 that this procedure underestimates the level density of an open-shell nucleus.

The main drawback of the Fermi-gas estimates is that these are based on an independentparticle assumption. Interactions between the particles are ignored and collective effects have to be introduced in a semi-empirical way. Likewise, the formalism developed so far assumes independent particles in a $\mathrm{HO}$ and thus suffers from the same problem. However, a simple generalization to an anisotropic HO leads to a formulation that accounts for an important collective effect, namely deformation. Although the problem can be formulated for a HO with complete anisotropy, assume by way of example that the quanta in two directions, say 1 and 2, have the same energy which is different from the 3-quantum energy, $\hbar \omega_{1}=\hbar \omega_{2} \equiv \hbar \omega_{12} \neq \hbar \omega_{3}$. This is appropriate for a deformed nucleus with axial symmetry; a prolate nucleus corresponds to $\omega_{12}>\omega_{3}$ while an oblate one has $\omega_{12}<\omega_{3}$. A Slater determinant is specified by the occupation numbers $k_{n_{1} n_{2} n_{3}}^{\sigma \tau}$ and its energy is

$$
E_{\mathrm{t}}=\left(\mathcal{N}_{12}+1\right) \hbar \omega_{12}+\left(\mathcal{N}_{3}+\frac{1}{2}\right) \hbar \omega_{3}
$$

where $\mathcal{N}_{12}$ is the number of quanta in the 1- and 2-directions, and $\mathcal{N}_{3}$ that of quanta in the 3 -direction. The number of distinct states for $A^{+}=N$ neutrons and $A^{-}=Z$ protons with $\mathcal{N}_{12}$ and $\mathcal{N}_{3}$ quanta, is determined by the solutions of

$$
\begin{aligned}
\sum_{n_{1} n_{2} n_{3}=0}^{\infty} \sum_{\sigma} k_{n_{1} n_{2} n_{3}}^{\sigma \tau} & =A^{\tau}, \quad \tau= \pm \\
\sum_{n_{1} n_{2} n_{3}=0}^{\infty} \sum_{\sigma \tau}\left(n_{1}+n_{2}\right) k_{n_{1} n_{2} n_{3}}^{\sigma \tau} & =\mathcal{N}_{12}, \\
\sum_{n_{1} n_{2} n_{3}=0}^{\infty} \sum_{\sigma \tau} n_{3} k_{n_{1} n_{2} n_{3}}^{\sigma \tau} & =\mathcal{N}_{3},
\end{aligned}
$$


and shall be denoted as $c_{3}\left(N, Z, \mathcal{N}_{12}, \mathcal{N}_{3}\right)$. The relevant recurrence relation in this case is

$$
\begin{aligned}
c_{3}\left(N, Z, \mathcal{N}_{12}, \mathcal{N}_{3}\right) & =\sum_{N^{\prime} Z^{\prime} \mathcal{N}_{12}^{\prime}} c_{2}\left(N^{\prime}, Z^{\prime}, \mathcal{N}_{12}^{\prime}\right) \\
& \times c_{3}\left(N-N^{\prime}, Z-Z^{\prime}, \mathcal{N}_{12}-\mathcal{N}_{12}^{\prime}, \mathcal{N}_{3}-N+N^{\prime}-Z+Z^{\prime}\right),
\end{aligned}
$$

and has the following boundary values:

$$
\begin{aligned}
c_{3}\left(N=0, Z=0, \mathcal{N}_{12}, \mathcal{N}_{3}\right) & =\delta_{\mathcal{N}_{12} 0} \delta_{\mathcal{N}_{3} 0} \\
c_{3}\left(N, Z, \mathcal{N}_{12}, \mathcal{N}_{3}\right) & =0, \quad \text { if } \quad \mathcal{N}_{12}+\mathcal{N}_{3}<\mathcal{M}_{3}^{N, Z}
\end{aligned}
$$

where $\mathcal{M}_{3}^{N, Z} \equiv \mathcal{M}_{3}^{N}+\mathcal{M}_{3}^{Z}$ denotes the minimum of quanta for $N$ neutrons and $Z$ protons.

The results of an illustrative calculation for the system corresponding to the nucleus ${ }^{28} \mathrm{Si}$ are shown in Fig. 3. Instead of the level density $\rho(E)$ itself, the cumulative number of levels $F(E)$ up to a certain energy $E$ is given, $F(E)=\int_{0}^{E} \rho\left(E^{\prime}\right) d E^{\prime}$. The energy scale is $\hbar \omega$ with $\omega$ the geometric mean of the frequencies, $\omega=\sqrt[3]{\omega_{12}^{2} \omega_{3}}$. The figure illustrates the important effect of deformation which in this particular nucleus (with many valence neutrons and protons) strongly reduces the level density with respect to the spherical result, shown for easy comparison in both the prolate and oblate case. There is little difference (for ${ }^{28} \mathrm{Si}$ ) between prolate and oblate 'normal' deformation $\left(\omega_{12} / \omega_{3} \approx \frac{3}{2}\right.$ and $\omega_{12} / \omega_{3} \approx \frac{2}{3}$, respectively, both in grey) but rather a lot between prolate and oblate 'super' deformation $\left(\omega_{12} / \omega_{3} \approx 2\right.$ and $\omega_{12} / \omega_{3} \approx \frac{1}{2}$, in black). The insets of the figure show cases of very small deformation (virtually identical for prolate and oblate) and compare them with the spherical staircase.

Possible applications of this formalism can be illustrated with a simple example. Figure 4 shows $F(E)$ for ${ }^{38}$ Ar. At low excitation energy, $F(E)$ can be obtained from counting known experimental levels [13]. Above $E \approx 7.5 \mathrm{MeV}$ too many levels have unknown spin $J$ and explicit counting becomes unreliable. Level densities at higher energies can be extracted from reaction work (see the compilation [14] and references therein) from where the limits on $F(E)$ shown in Fig. 4 can be derived. The calculation of the theoretical level density proceeds as follows. It first requires the determination of the oscillator frequencies which can be taken from (see Chap. 5 of $[15]) \omega_{12}=\omega\left(1+\frac{1}{3} \delta\right)$ and $\omega_{3}=\omega\left(1-\frac{2}{3} \delta\right)$ where $\hbar \omega=41 A^{-1 / 3}$ 
$\mathrm{MeV}$ sets the energy scale and $\delta=\sqrt{45 / 16 \pi} \beta_{2}$, with $\beta_{2}$ the quadrupole deformation. For a given $\beta_{2}$ one then looks for the values of $\mathcal{N}_{12}$ and $\mathcal{N}_{3}$ that minimize the energy (13) and for which the corresponding number of configurations $c_{3}\left(N, Z, \mathcal{N}_{12}, \mathcal{N}_{3}\right)$ is non-zero. This defines the ground-state configurations. The number of configurations at non-zero excitation energy is obtained by increasing the number of quanta or by transforming 3-quanta into 12 quanta if $\omega_{12}>\omega_{3}$ or vice versa if $\omega_{12}<\omega_{3}$. The results of such a calculation at two different deformations, $\beta_{2}=-0.2$ and 0.2 , are shown in Fig. 4 in grey. Given the simplicity of the approach, the agreement can be called satisfactory. A possible source of uncertainty is the choice of the deformation parameter. The nucleus ${ }^{38} \mathrm{Ar}$ is near-spherical, $\beta_{2} \approx 0$, but probably a larger value of $\beta_{2}$ is more appropriate at higher excitation energy. It should be noted that the agreement is not always as gratifying as it is in ${ }^{38} \mathrm{Ar}$ : If one repeats a similar exercise for $f_{7 / 2}$ nuclei with known level densities at high energy such as Fe or Ni, the calculation overestimates the observed densities. This is a consequence of the propinquity of the $N, Z=28$ shell closure which is absent from the HO.

The applications of the recursive formalism discussed in this Letter have centered around the harmonic oscillator mainly because of the intrinsic interest of such a system and also because it can readily accommodate deformation. It is, however, easy to generalize the present recursive method to a system of fermions distributed over a set of single-particle levels with arbitrary energies and degeneracies. Thus, applications of the type reported in [16], based on a recursive technique for partitions functions, can also be carried out with the current formalism. The use of realistic single-particle energies in combination with deformation effects and a reliable estimate of the number of spurious states should lead to fruitful applications of the present formalism in the domain of nuclear level densities.

I wish to thank Gérard Auger for fruitful discussions and Alex Brown for his suggestion concerning spurious states. 


\section{REFERENCES}

[1] H.A. Bethe, Phys. Rev. 50, 332 (1936); Rev. Mod. Phys. 9, 69 (1937).

[2] A. Bohr and B.R. Mottelson, Nuclear Structure. I Single-particle motion (Benjamin, New York, 1969).

[3] J. Bardeen and E. Feenberg, Phys. Rev. 54, 809 (1938).

L. Motz and E. Feenberg, Phys. Rev. 54, 1055 (1938).

[4] C. Bloch, Phys. Rev. 93, 1094 (1954).

[5] N. Rosenzweig, Phys. Rev. 108, 817 (1957).

[6] V. Paar and R. Pezer, Phys. Rev. C 55, R1637 (1997).

[7] A.P. Zuker, Phys. Rev. C 64, 021303(R) (2001).

[8] T. von Egidy, H.H. Schmidt, and A.N. Behkami, Nucl. Phys. A 481, 189 (1988).

[9] T. Rauscher, F.-K. Thielemann, and K.-L. Kratz, Phys. Rev. C 56, 1613 (1997).

[10] J.B. French and V.K.B. Kota, Annu. Rev. Nucl. Part. Sci. 32, 35 (1982).

[11] H. Nakada and Y. Alhassid, Phys. Rev. Lett. 79, 2939 (1997).

W.E. Ormand, Phys. Rev. C 56, R1678 (1997).

K. Langanke, Phys. Lett. B 438, 235 (1998).

[12] R.D. Lawson, Theory of the Nuclear Shell Model (Clarendon Press, Oxford, 1980).

[13] R.B. Firestone, Table of Isotopes (Wiley-Interscience, New York, 1996).

[14] A.S. Iljinov et al., Nucl. Phys. A 543, 517 (1992).

[15] A. Bohr and B.R. Mottelson, Nuclear Structure. II Nuclear Deformations (Benjamin, New York, 1975).

[16] S. Pratt, Phys. Rev. Lett. 84, 4255 (2000). 


\section{FIGURES}

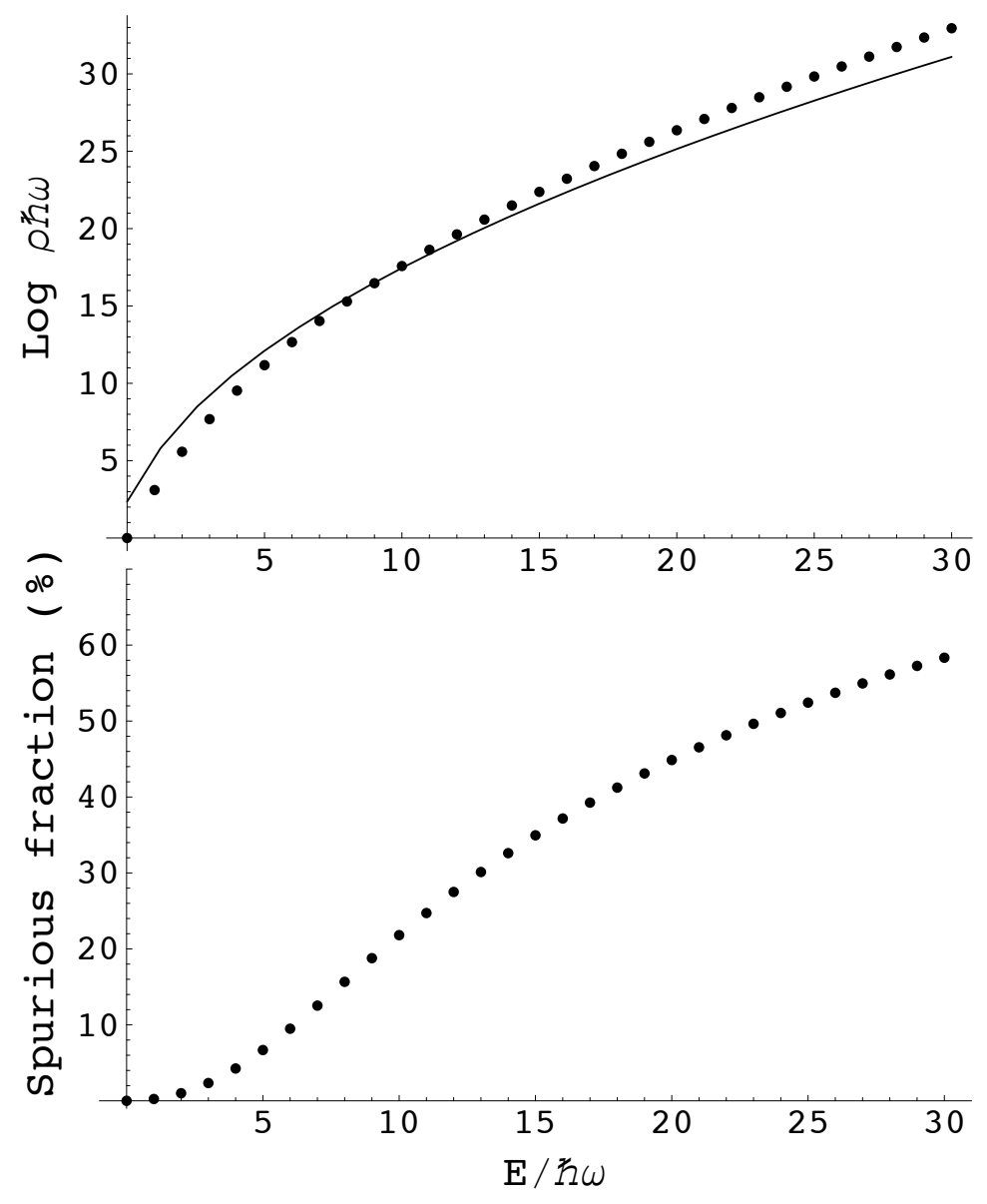

FIG. 1. Top: The level density $\rho(A, E)$ for $A=70 s=\frac{1}{2}$ particles. The full curve is the level density computed from Bethe's one-component Fermi gas formula, and the dots represent $c_{3}(A, \mathcal{N})$. Bottom: The number of spurious states as a fraction of the total number of states. 


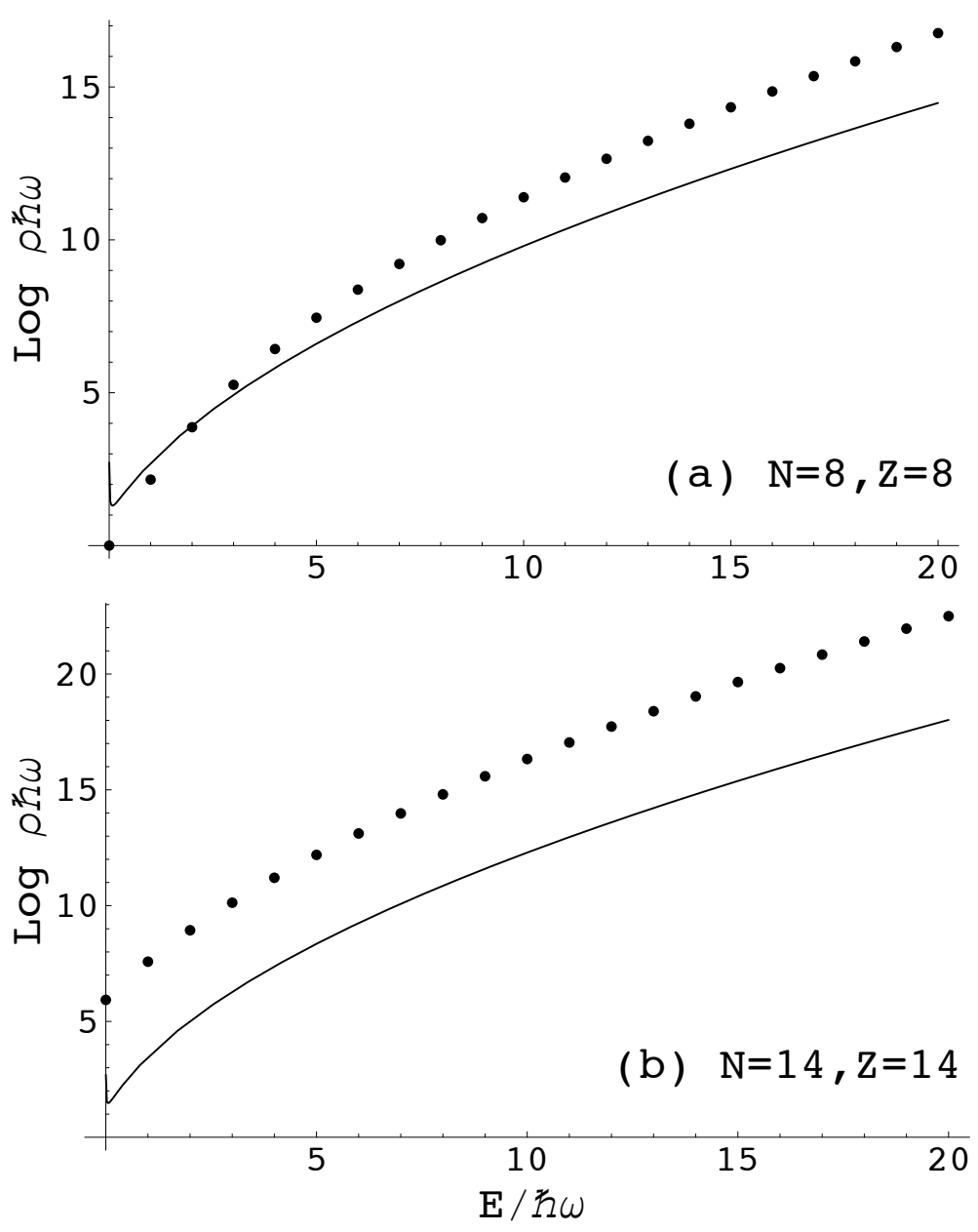

FIG. 2. The level density $\rho(N, Z, E)$ for (a) $N=Z=8$ and (b) $N=Z=14$. The full curves are the level densities computed from Bethe's two-component Fermi-gas formula and the dots represent $c_{3}(N, Z, \mathcal{N})$. 

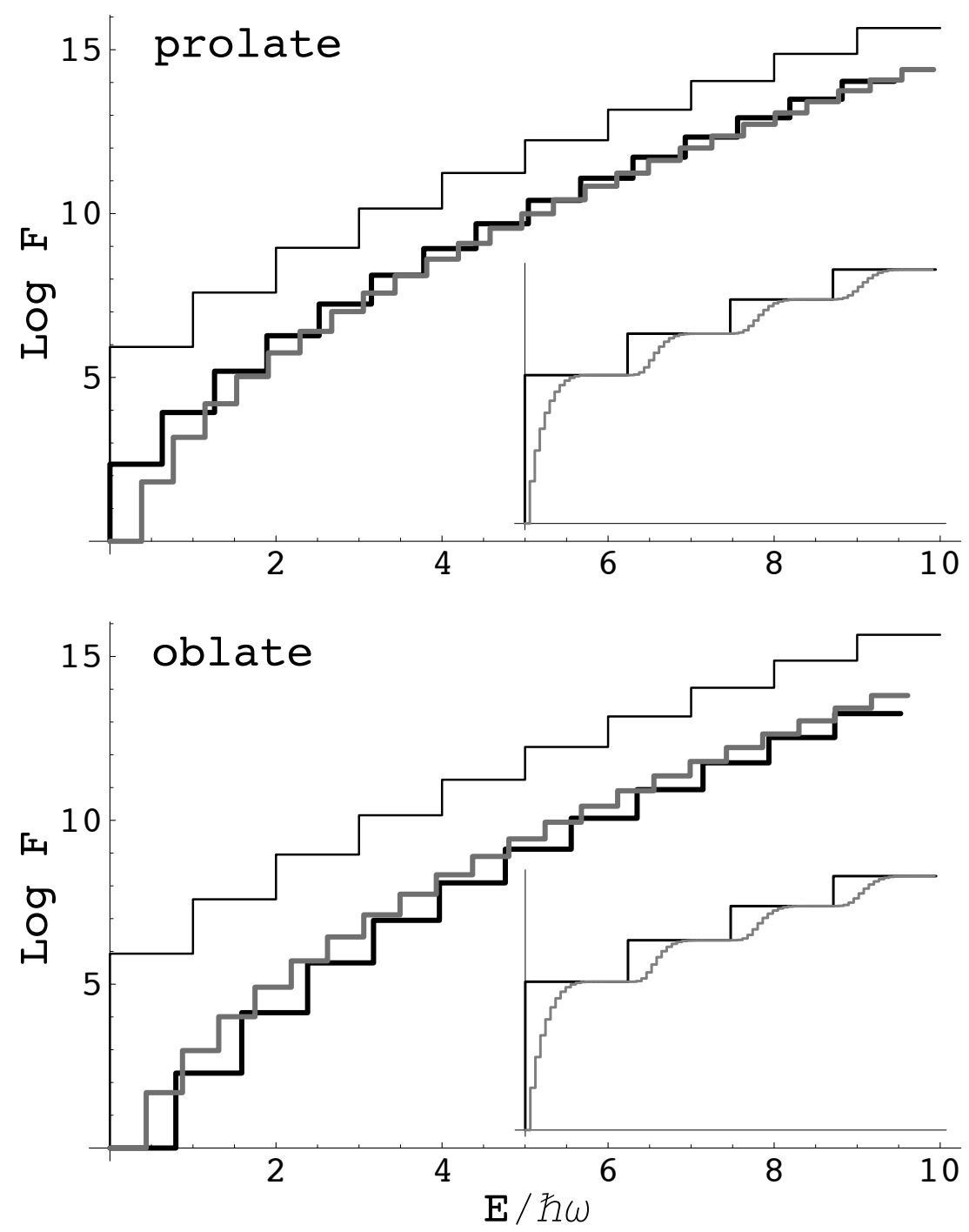

FIG. 3. The number of levels $F(N, Z, E)$ for $N=Z=14$ for prolate and oblate deformation. The thick grey lines are for 'normal' deformation (axes ratio $2 \div 3$ ) while the thick black lines are for 'super' deformation (axes ratio $1 \div 2$ ). The thin line gives the spherical result. The insets show cases of very small deformation (prolate and oblate) as compared with the spherical staircase. 


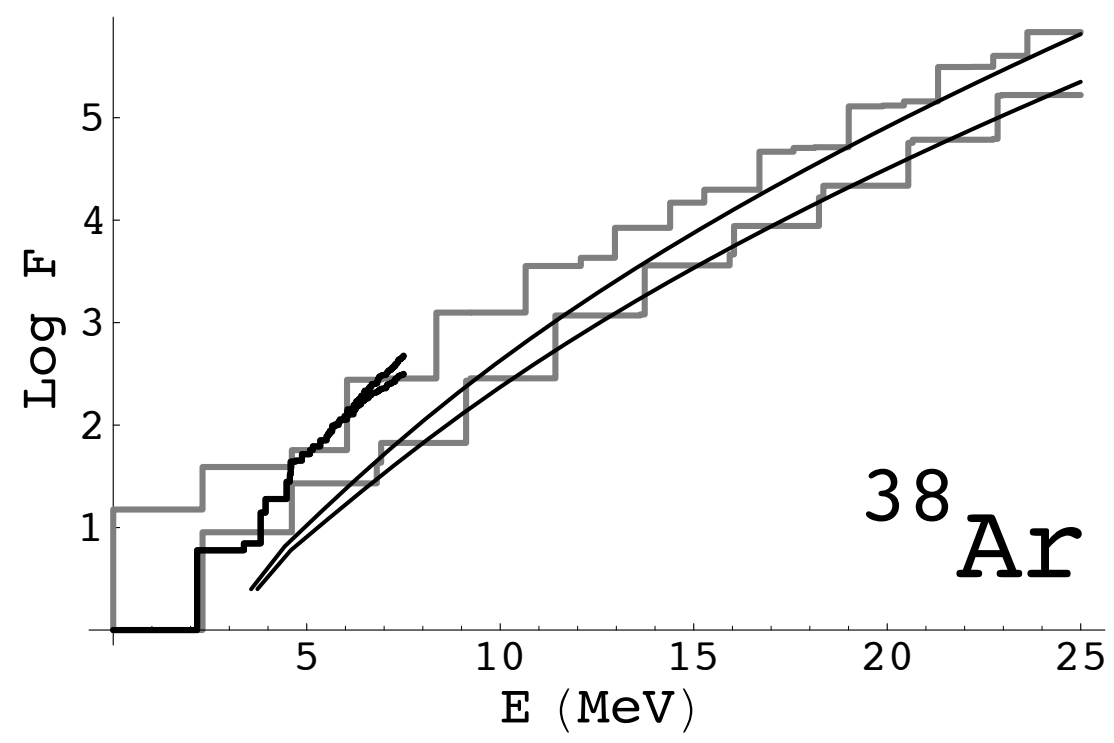

FIG. 4. The number of levels $F(E)$ in ${ }^{38}$ Ar. The black jagged lines are lower and upper limits obtained from counting levels. The smooth curves are the lower and upper limits for $F(E)$ obtained by fitting Bethe's two-component Fermi-gas formula [with back shift, i.e. replace $E$ by $E+\Delta$ in Eq. (12)] to the experimental densities from [14]. The grey lines are calculated following the procedure explained in the text for two different deformations $\beta_{2}=-0.2$ (lower) and $\beta_{2}=0.2$ (upper). 